Check for updates

Cite this: J. Mater. Chem. B, 2018, 6,6153

Received 12th June 2018,

Accepted 21st August 2018

DOI: $10.1039 / c 8 t b 01544 a$

rsc.li/materials-b

\section{Rapid bioinspired mineralization using cell membrane nanofragments and alkaline milieu $\dagger$}

\author{
Emilio Satoshi Hara, (D) a Masahiro Okada, (D) a Takuo Kuboki, (D) ${ }^{\text {b }}$ \\ Takayoshi Nakano (D) ${ }^{c}$ and Takuya Matsumoto (D)*a
}

\begin{abstract}
Bone is a sophisticated organic-inorganic hybrid material, whose formation involves a complex spatiotemporal sequence of events regulated by the cells. A deeper understanding of the mechanisms behind bone mineralization at different size scales, and using a multidisciplinary approach, may uncover novel pathways for the design and fabrication of functional bone tissue in vitro. The objectives of this study were first to investigate the environmental factors that prime initial mineralization using the secondary ossification center as an in vivo model, and then to apply the obtained knowledge for rapid in vitro synthesis of bone-like tissue. First, the direct and robust measurement of $\mathrm{pH}$ showed that femur epiphysis is alkaline $(\mathrm{pH} \cong 8.5)$ at the initial mineral stage at post-natal day 6 . We showed that the alkaline milieu is decisive not only for alkaline phosphatase activity, which precedes mineral formation at P6, but also for determining initial mineral precipitation and spherical morphology. Next, engineering approaches were used to synthesize bone-like tissue based on alkaline milieu and artificial chondrocyte membrane nanofragments, previously shown to be the nucleation site for mineral formation. Interestingly, mineralization using artificial cell membrane nanofragments was achieved in just 1 day. Finally, ex vivo culture of femur epiphysis in alkaline $\mathrm{pH}$ strongly induced chondrocyte burst, which was previously shown to be the origin of chondrocyte membrane nanofragments, and also enhanced mineral formation. Taken together, these findings not only shed more light on the microenvironmental conditions that prime initial bone formation in vivo, but they also show that alkaline milieu can be used as an important factor for enhancing methods for in vitro synthesis of bone tissue.
\end{abstract}

\section{Introduction}

Biomineralization involves a complex spatio-temporal sequence of events regulated by the cells that build sophisticated organic-inorganic hybrid materials such as bone and teeth. ${ }^{1,2}$ The highly sophisticated composition and functional adaptability of bones and teeth have inspired chemists and materials scientists to mimic such structures and properties for diverse applications, including tissue engineering and regenerative medicine. In the tissue engineering field, in vitro bone tissue synthesis has been partly achieved by using cells,

\footnotetext{
${ }^{a}$ Department of Biomaterials, Okayama University Graduate School of Medicine, Dentistry and Pharmaceutical Sciences, 2-5-1 Shikata-cho, Kita-ku, Okayama-shi, Okayama-ken, 700-8525, Okayama, Japan. E-mail: tmatsu@md.okayama-u.ac.jp; Fax: +81 86235 6669; Tel: +81 862356665

${ }^{b}$ Department of Oral Rehabilitation and Regenerative Medicine, Okayama University Graduate School of Medicine, Dentistry and Pharmaceutical Sciences, Okayama, Japan

${ }^{c}$ Division of Materials and Manufacturing Science, Graduate School of Engineering, Osaka University, Osaka, Japan

$\dagger$ Electronic supplementary information (ESI) available. See DOI: 10.1039/ c8tb01544a
}

biomaterials and growth factors, ${ }^{3-5}$ or by using only materials such as hydrogels and inorganic solutions. ${ }^{6-8}$ However, further improvement in the methods to achieve real bone tissue engineering with more precise manipulation of bone tissue growth and maturation is still necessary. Therefore, a deeper understanding of the mechanisms behind bone mineralization in vivo at different size scales, and integrating biology, materials science and chemistry, may drive novel pathways for the development of novel methods and techniques, and for the design and fabrication of new functional biomaterials for bone tissue synthesis and regeneration.

From a biological viewpoint, animal models with complete or tissue-specific gene deletion have been used to understand the importance of cell activities in bone formation. For instance, transcription factors (e.g., Runx2 and $O s x),{ }^{9,10}$ intracellular signaling molecules (e.g., $\beta$-catenin $)^{11}$ and extracellular proteins (e.g., Wnts, BMPs, and FGFs) ${ }^{12,13}$ have been shown to be crucial in determining cell differentiation and cell-cell communication towards bone formation. Along with cell differentiation stages (e.g., pre-osteoblats, mature osteoblasts, proliferating chondrocytes, and hypertrophic chondrocytes), ultrastructural analysis of the calcifying tissue showed that cell-secreted matrix vesicles ${ }^{14-16}$ 
and chondrocyte death-originated membrane nanofragments ${ }^{1,17}$ were the nucleation site for mineral formation in intramembranous and endochondral ossification, respectively. This evidence was further confirmed by analysis of mineral formation from the chemistry and materials science approach, showing that cell membrane nanofragments, collagens and non-collagenous proteins can be the nucleation sites for the initial mineral deposition. ${ }^{1,18-20}$

Nevertheless, besides the cell differentiation stage, which determines the overall bone formation process, and composition of the matrix (i.e., scaffold), where the minerals will in fact be deposited, the physico-chemical characteristics of the microenvironment, including the electrical charge of proteins and ion concentrations, are of critical importance for priming initial mineralization. For instance, bone formation is known to be dependent on several enzymes, such as tissue non-specific alkaline phosphatase (TNAP) and phosphoethanolamine/ phosphocholine phosphatase (PHOSPHO-1), which have been regarded as the major phosphatases required for mineral deposition. ${ }^{21}$ The optimal $\mathrm{pH}$ for alkaline phosphatase has been reported to be from 8.5 to $9.5 .^{22}$ Therefore, a possible alkaline environment in promoting calcification in calcifying tissue has been hypothesized. Nevertheless, confirmation of an alkaline environment in calcifying tissues has been challenging due to the complexity of preparation of intact samples as well as due to the relatively inaccurate techniques for analysis of $\mathrm{pH}$. A previous investigation measured $\mathrm{pH}$ in calcified femur epiphysis and found it to be approximately 7.5. However, samples were kept in Lock's solution, which could immediately affect the environmental $\mathrm{pH}^{23}$ Therefore, more robust models and techniques for $\mathrm{pH}$ measurement in live tissues are necessary to elucidate the environmental conditions optimal for bone formation.

In a previous study, we demonstrated that initial mineral formation in mouse femur epiphysis (secondary ossification center) initiates at post-natal day 5 (P5). ${ }^{1}$ It is of note that the initial mineral formation until P6 was based exclusively on chondrocyte-derived membrane nanofragments, i.e., there were no other cell types (e.g., osteoblasts and endothelial cells) inside the epiphysis. ${ }^{1,17}$ This makes epiphysis a robust and easy-handling in vivo model for understanding the microenvironmental conditions associated with initial bone formation. In this study, first, alkaline phosphatase activity was detected one day prior to mineralization, and subsequent analysis showed that the $\mathrm{pH}$ at epiphysis is alkaline. From the chemistry perspective, the effects of alkaline $\mathrm{pH}$ on mineral nucleation and morphology were also demonstrated. Finally, we also demonstrated that alkaline $\mathrm{pH}$ enhances matrix synthesis by chondrocytes, and induces chondrocyte burst, which was associated with space making for mineral expansion. ${ }^{17}$

\section{Materials and methods}

\section{Animals, histological analysis and immunohistochemistry}

New-born Balb/c mice from post-natal day 2 (P2) to P7 were used in the experiments according to the Guidelines for Animal Research of Okayama University. The Animal Care and Use
Committee of Okayama University approved the research protocols (OKU-2014283 and OKU-2015542).

Femur epiphyses were isolated and immediately embedded in cryosection medium (Section-lab, Hiroshima, Japan) for cryosectioning. For histological staining, serial cryosections of $10 \mu \mathrm{m}$ in thickness were fixed in $4 \%$ paraformaldehyde (PFA) for 2 minutes, and stained with alizarin red $\mathrm{S}$ for $3 \mathrm{~min}$ for identification of the mineralized area, or with 5-bromo-4chloro-3-indolyl phosphate/nitro blue tetrazolium (BCIP/NBT, Roche, Basel, Switzerland) for $3 \mathrm{~min}$ for analysis of alkaline phosphatase activity in tissue sections. Samples were then washed and observed under a microscope (Biozero BZ-X700, Keyence, Osaka, Japan).

For immunohistochemical analysis, serial cryosections of $10 \mu \mathrm{m}$ of epiphysis samples were fixed with PFA, washed in PBS, and blocked with $5 \%$ goat serum and then immunolabeled with primary antibody or the isotype-matched IgG antibody at $4{ }^{\circ} \mathrm{C}$ overnight. $^{24}$ Antibody for carbonic anhydrase II (ab191343) was purchased from Abcam (Cambridge, UK). The target proteins were visualized with secondary antibody conjugated with Alexa Fluor 488 or 647 (Life Technologies, Gaithersburg, MD, USA) under a fluorescence confocal microscope (LSM780, Carl Zeiss MicroImaging, Jena, Germany). Cell nuclei were stained with Hoechst-3334 (Life Technologies).

\section{Fourier transform infrared (FT-IR) spectroscopic analysis}

Epiphyses were isolated from newborn mice at P4 to P7 and sliced transversally. Attenuated total reflectance (ATR) FT-IR spectra were recorded after pressing the epiphysis slices on a ZnSe prism equipped on an IRAffinity-1S (Shimadzu, Kyoto, Japan) at $4 \mathrm{~cm}^{-1}$ resolution with 32 scans at room temperature around $25{ }^{\circ} \mathrm{C}^{25}$ The spectra were analyzed using peaks indicating phosphate (900 to $1200 \mathrm{~cm}^{-1}$ ) and the organic part of the cartilage collagenous matrix was assessed using the amide I peak (1584 to $1720 \mathrm{~cm}^{-1}$ ), according to previous reports. ${ }^{26,27}$

\section{Real-time reverse transcription polymerase chain reaction (RT-PCR) analysis}

Total RNA from P2 to P7 epiphyses was extracted with trizol (Life Technologies) and after sonication, RNA was purified using a Purelink (Life Technologies) kit, according to the manufacturer's instructions. Real-time reverse-transcription polymerase chain reaction (RT-PCR) was used for mRNA quantification as described previously. ${ }^{28}$ Primer sequences for $\mathrm{Alpl}$ are: sense: $5^{\prime}$-GCTCTCCCTACCGACCCTGTTC-3' and antisense: $5^{\prime}$-TGCTGGAAGTTGCCTGGACCTC-3' . Primer sequences for Gapdh are: sense $5^{\prime}$-TGACAATGAATACGGCTACAG- $3^{\prime}$ and antisense 5'-GGAGATGCTCAGTGTTGG-3'. The levels of Alpl mRNA were normalized to that of the reference gene Gapdh.

\section{In vivo $\mathrm{pH}$ measurement}

Muscles and epiphyses at P3 and P6 (cross-sectional slices) were harvested and immediately placed onto a fluorescence $\mathrm{pH}$ sensor sheet (SF-HP5R VisiSens, PreSens Precision Sensing $\mathrm{GmbH}$, Regensburg, Germany), which has a precision of \pm 0.01 $\mathrm{pH}$ at $\mathrm{pH}=7$, according to the manufacturer's protocol. The surface 
of the $\mathrm{pH}$ sensor sheet is covered with a sensor film, which translates the spatial $\mathrm{pH}$ content into a fluorescent light signal. After the samples were placed onto the sheet, it was then immediately observed under a fluorescence confocal microscope (LSM780, Carl Zeiss) at $25{ }^{\circ} \mathrm{C}$. The green fluorescent signal was recorded from at least 2 different regions per sample. For analysis of the fluorescence intensity, captured images were converted into greyscale images by using Adobe Photoshop 12 (Adobe Systems, San Jose, CA, USA), and the mean grey value was obtained by using Image software (NIH, Bethesda, MD, USA) to determine the fluorescence intensity. PBS solutions of $\mathrm{pH} 6.5, \mathrm{pH} 7.5$ and $\mathrm{pH} 8.5$ were used as standards to determine the $\mathrm{pH}$ of the tissues. Standard PBS solutions were titrated with $\mathrm{NaOH}$ or $\mathrm{HCl}$ for the desired $\mathrm{pH}$.

\section{Field emission scanning electron microscopy (FE-SEM) and energy-dispersive X-ray spectroscopy (EDS) analysis}

For FE-SEM observation, P6 epiphyses were collected and immediately treated with sodium hypochlorite for $10 \mathrm{~min}$ to remove all organic materials. The minerals were then washed, dehydrated with ethanol, and then fixed onto an aluminum holder and submitted to osmium coating (Neoc-STB, Meiwafosis, Tokyo, Japan) at an electrical discharge current of $10 \mathrm{~mA}$ and a degree of vacuum of $10 \mathrm{~Pa}$ for $20 \mathrm{~s}$, before FE-SEM observation (FE-SEM; JSM-6701F, JEOL), operated at $5 \mathrm{kV}$ at $10 \mathrm{~mA}$, and equipped with an EDS detector (Apollo XV, EDAX, Mahwah, NJ, USA).

\section{In vitro mineral precipitation assay}

PBS solutions containing $1.37 \mathrm{M} \mathrm{NaCl}, 2.7 \mathrm{mM} \mathrm{KCl}, 10 \mathrm{mM}$ $\mathrm{Na}_{2} \mathrm{HPO}_{4} \cdot 12 \mathrm{H}_{2} \mathrm{O}$ and $1.8 \mathrm{mM} \mathrm{KH} \mathrm{PO}_{4}$ were titrated with $\mathrm{HCl}$ or $\mathrm{NaOH}$ to obtain solutions with different pHs $(6.5,7.5,8.5$ and 9.5). $\mathrm{CaCl}_{2}$ solutions of different osmolality $(0.5 \mathrm{mM}$, $1 \mathrm{mM}$ and $2 \mathrm{mM}$ ) were prepared and slowly added into PBS solutions of different $\mathrm{pHs}$, at room temperature, without $\mathrm{pH}$ control. Precipitated minerals were immediately centrifugally washed 3 times and vacuum dried for weight measurement using a weighing balance with milligram precision. Precipitated mineral amounts below 1 milligram could not be measured. Mineral characterization was performed by X-ray diffraction (XRD) measurements on an instrument (RINT2500HF; Rigaku Corp., Tokyo, Japan) equipped with a $\mathrm{Cu}-\mathrm{K} \alpha$ radiation source.

\section{In vitro mineralization in collagen gel}

A gel diffusion system was prepared based on a previously reported model. ${ }^{29}$ In this experiment, a collagen gel [Cellmatrix type I-A (Nitta Gelatin Inc., Osaka, Japan) mixed with a neutralizing buffer: $0.05 \mathrm{~N} \mathrm{NaOH} / 2.2 \% \mathrm{NaHCO}_{3} / 200 \mathrm{mM}$ HEPES] was prepared and poured into the opened bottom of a $50 \mathrm{~mL}$ tube, sealed with paraffin tape (Parafilm, Bemis NA, Neenah, WI, USA), and kept in an incubator at $37{ }^{\circ} \mathrm{C}$ for $30 \mathrm{~min}$ to facilitate gelation. The calcium $\left[100 \mathrm{mM}\left(\mathrm{CH}_{3} \mathrm{COO}\right)_{2} \mathrm{Ca} \cdot \mathrm{H}_{2} \mathrm{O}\right]$ and phosphate $\left(60 \mathrm{mM} \mathrm{NH}_{4} \mathrm{H}_{2} \mathrm{PO}_{4}\right)$ were then poured into the opposite sides of the collagen gel and left for $30 \mathrm{~min}$. The gel was then fixed in 4\% PFA, and the minerals formed in the center of the gel were observed using SEM after osmium coating. To analyze the effect of different pHs on the shape of the precipitated minerals, calcium and phosphate solutions were titrated with $\mathrm{HCl}$ or $\mathrm{NaOH}$ to obtain solutions with $\mathrm{pH}$ 6.5, $\mathrm{pH} 7.5$ or $\mathrm{pH} 8.5$ before pouring into the gel diffusion system.

\section{Effect of pH on extracellular matrix synthesis}

ATDC5 prechondrogenic cells were maintained in Dulbecco's Modified Eagle Medium and Ham's F-12 medium (DMEM/F-12; Wako Pure Chemical Industries, Osaka, Japan) containing 10\% fetal bovine serum (FBS; Life Technologies) and 1\% penicillin and streptomycin (Sigma, St Louis, MO, USA). Cells were cultured until confluency, trypsinized and centrifuged. Cells were then resuspended with culture medium at a concentration of $2 \times 10^{7}$ cells per $\mathrm{mL}$ and seeded in $10 \mu \mathrm{L}$ micromasses. ${ }^{24}$ Micromasses were then cultured in chondrogenic media with different pHs for 3 days. Chondrogenic medium consisted of DMEM/F12 supplemented with $6 \mu \mathrm{g} \mathrm{mL}{ }^{-1}$ insulin, transferrin and selenous acid (ITS solution, BD, Bedford, MA, USA), L-ascorbic acid

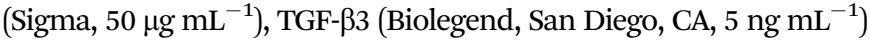
and fluocinolone acetonide $\left(100 \mathrm{ng} \mathrm{mL}^{-1}\right.$, Tokyo Chemical Industry). The chondrogenic media were titrated with $\mathrm{NaOH}$ or $\mathrm{HCl}$ to get the desired $\mathrm{pH}$.

For alcian blue staining of glycosaminoglycans, micromasses were fixed with $4 \%$ PFA, washed, and then stained with alcian blue staining solution pH 2.5 for cytology (Muto Pure Chemicals Co., LTD., Tokyo, Japan). Samples were then washed thoroughly to remove excess stain and then photographed under a light microscope (CKX41, Olympus). ${ }^{30}$

\section{Effect of pH on chondrocyte burst and mineral formation}

To analyze the effect of $\mathrm{pH}$ on chondrocyte burst, DMEM/F12 with $\mathrm{pH} 6.5$, $\mathrm{pH} 7.5$ and $\mathrm{pH} 8.5$ was prepared by titration with $\mathrm{NaOH}$ or $\mathrm{HCl}$. Epiphysis cartilage slices were then incubated in each medium type and observed with a $40 \times$ optical zoom lens under a microscope (Nikon Eclipse Ti, Tokyo, Japan) at $37{ }^{\circ} \mathrm{C}$ with $5 \% \mathrm{CO}_{2}$ in air for $60 \mathrm{~min}$. Time-lapse images were then processed to generate a movie using ImageJ software, and then analyzed for the number of chondrocyte bursts. Mounted videos were used for qualitative and quantitative measurements of chondrocyte burst per $0.35 \times 10^{5} \mu \mathrm{m}^{2}$. Chondrocyte burst was defined as a change in cellular membrane conformation followed by a decrease in cell size, as described previously. ${ }^{17}$

For analysis of the effect of $\mathrm{pH}$ on mineral formation in epiphysis after induction of chondrocyte burst, isolated P6 femur epiphyses were incubated in DMEM/F12 with different pHs (6.5, 7.5 and 8.5) for $24 \mathrm{~h}$, and then in mineralization-inducing medium (DMEM/F12 supplemented with $\beta$-glycerophosphate) for an additional 2 days. After incubation in mineralization-inducing medium, samples were fixed with $4 \%$ PFA and submitted to bone volume analysis by micro-CT.

\section{X-ray micro computed tomography (micro-CT)}

Micro-CT images of the collected epiphysis were obtained using a SkyScan 1174 compact micro-CT (SkyScan, Aartselaar, Belgium). CT scans were performed at a resolution of $6.4 \mu \mathrm{m}$, and sections were reconstructed to produce the final 3D images using Nrecon 
and CTVol SkyScan software. Bone volume was analyzed under the same parameters using CTAn SkyScan software.

\section{Statistical analysis}

Analysis of the differences between groups was performed with the unpaired Student's $t$-test or one-way ANOVA followed by a Tukey post hoc correction test when appropriate. All statistical analyses were performed with StatView-5 software (SAS Institute Inc. Cary, NC).

\section{Results}

\section{Alkaline environment primes alkaline phosphatase activity before mineralization}

To identify the initial mineral deposition in the cartilage epiphysis of mouse femur, we first performed histological staining of epiphyses from post-natal day 5 (P5) to P7, as reported previously. ${ }^{1}$ Alizarin red staining shows the initial minerals in the medial side of the epiphysis at P6 (Fig. 1A). It is of note that alkaline phosphatase activity was detected in hypertrophic chondrocytes in the medial side of P5 epiphysis, which corresponds to the exact site where minerals would be formed at P6 epiphysis. Accordingly, the increase in the area showing positive staining for phosphatase activity at P6 corresponded to the mineralized area at $\mathrm{P} 7$, indicating that alkaline phosphatase activity anticipated the initial mineral formation, possibly by releasing phosphate ions from organic molecules such as phospholipids and phosphoproteins. In fact, the phosphate amount in the tissue, measured by FITR spectroscopy, gradually increased from P4 to P7 (Fig. 1B). Among several alkaline phosphatases, TNAP has been reported to be one of the major phosphatases associated with bone formation. ${ }^{21}$ Thus, we investigated the mRNA levels of Alpl by real time RT-PCR, and found that the increase in Alpl matches the phosphatase activity from P5 onward (Fig. 1C). Together, these results indicate that the activity of TNAP, and possibly of other phosphatases, is necessary for phosphate ion release to promote initial mineral formation in P6 femur epiphysis.

Mammalian TNAP is anchored to the plasma membrane by glycosylphosphatidylinositol and has an optimal $\mathrm{p} K_{\mathrm{a}}$ between 8.5 and 9.5. ${ }^{22}$ Thus, we attempted to measure the microenvironmental $\mathrm{pH}$ using a $\mathrm{pH}$ sensor sheet, and interestingly, we found that the $\mathrm{pH}$ of $\mathrm{P} 6$ femoral muscle and epiphysis was 7.32 and 8.61, respectively (Fig. 2). It is worth noting that until P6, the epiphysis contains no blood cells or any other cell types in its inner region. ${ }^{1}$ Therefore, until P6, the measurement of $\mathrm{pH}$ of the epiphysis is relatively easy and reliable compared to highly vascularized tissues or hard tissues, such as enamel or mature bone. These results indicate that the alkaline microenvironment primes the alkaline phosphatase activity for initial mineral deposition.

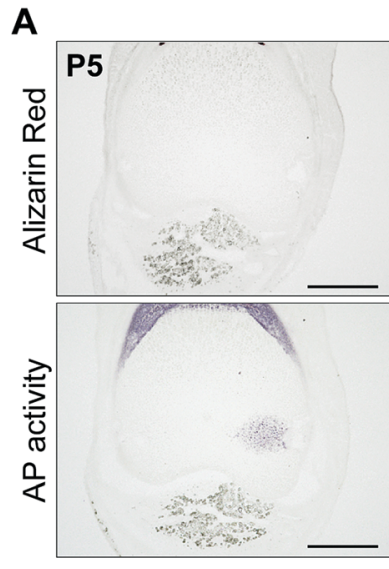

B

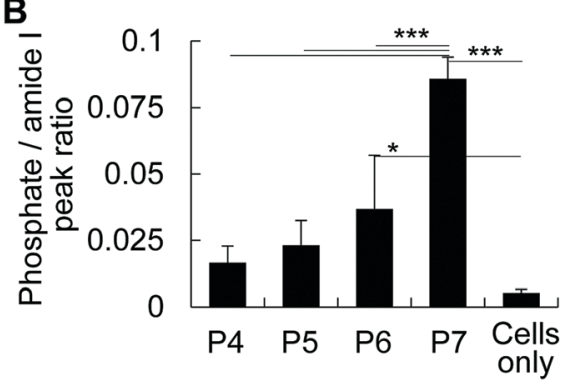

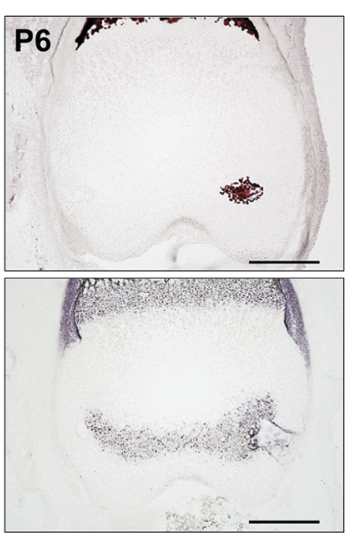

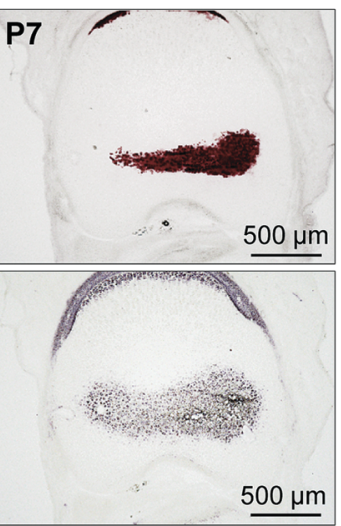

C

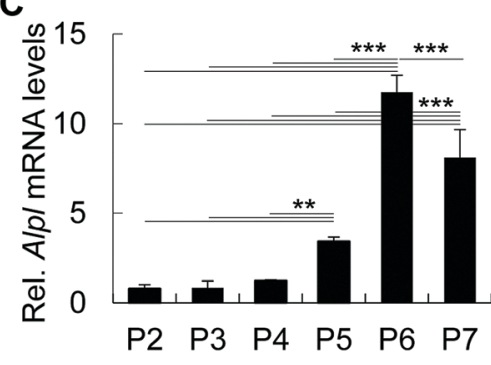

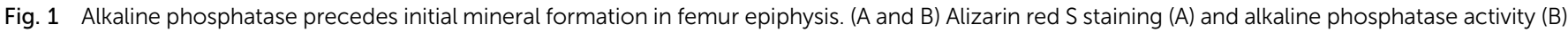

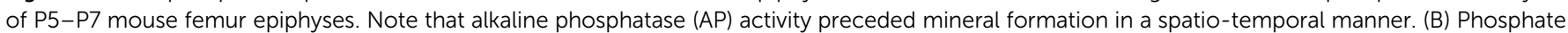

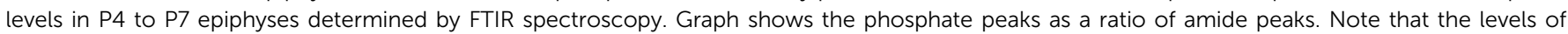

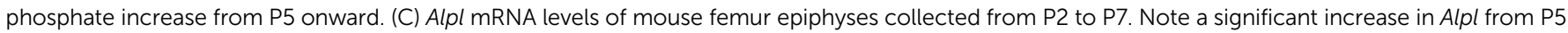

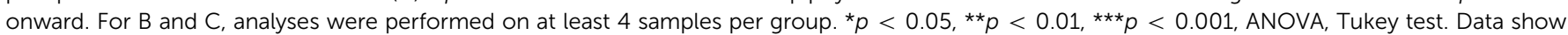
mean \pm SD of at least three different samples. 

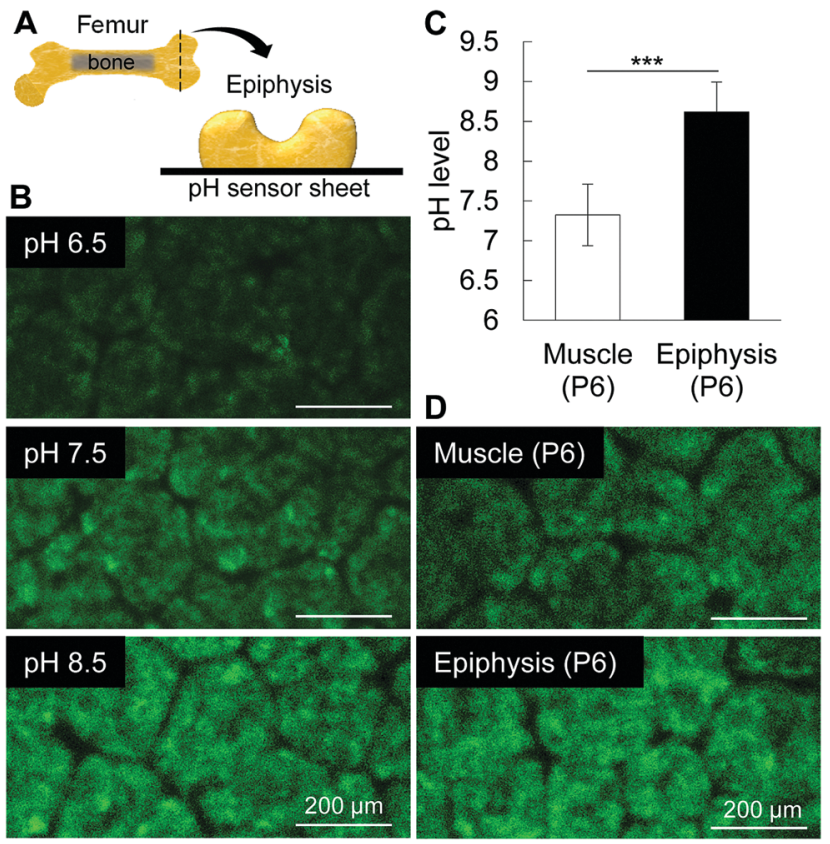

Fig. $2 \mathrm{pH}$ measurement in femur epiphyses. (A) Schematic design of the femur epiphysis in new born mice. The epiphysis was sliced transversally and immediately placed onto the $\mathrm{pH}$ sensor sheet. (B) Images show the fluorescence intensities of the standard solutions $(\mathrm{pH} \mathrm{6.5,7.5} \mathrm{and} \mathrm{8.5),}$ muscle and epiphysis. ( $C$ and D) Graph and images show that $\mathrm{pH}$ in muscle is neutral, near 7.5, whereas that of epiphysis is alkaline, near 8.5. The green patterns seen in the figures are originally of the $\mathrm{pH}$ sensor. The patterns do not correspond to any part of the cartilage tissue. ${ }^{* \star \star} p<0.001$, Students' $t$-test. Data show mean \pm SD of at least 4 different samples.

\section{Alkaline pH facilitates calcium phosphate nucleation and determines mineral shape}

Taking into consideration that the microenvironment in P6 epiphysis is rich in phosphate, we then investigated the effect of $\mathrm{pH}$ on the minimum amount of calcium necessary for mineral precipitation. As shown in Fig. 3, we prepared PBS solutions at different $\mathrm{pHs}$ and added calcium solution $\left(\mathrm{CaCl}_{2}\right)$ to final concentrations of $0.5 \mathrm{mM}$ to $2 \mathrm{mM}$. Interestingly, at pH 8.5, the minimum amount of calcium necessary for mineral precipitation was markedly lower $(1 \mathrm{mM})$ compared to that pH 7.5 (2 mM), as shown in Fig. 3B. At pH 9.5, the minimum calcium amount for mineral deposition was even lower $(0.5 \mathrm{mM})$.
At $\mathrm{pH}$ 6.5, there was no mineral formation even at high concentrations of calcium $(2 \mathrm{mM})$. XRD analysis identified the precipitated minerals (products) to be HAp under all $\mathrm{pH}$ conditions (Fig. 3C).

Next, we analyzed the effect of alkaline $\mathrm{pH}$ on mineral growth and morphology. We first performed SEM observation of in vivo minerals obtained from P6 epiphysis, following sodium hypochlorite treatment to remove all organic materials. The results revealed that the initial minerals in P6 epiphysis presented a spherical shape (Fig. 4A-C). Next, we performed mineralization in a gel diffusion system using collagen gel and calcium and phosphate solutions at different pHs (Fig. 4D). As shown in Fig. 4E, only at $\mathrm{pH} 8.5$ did the minerals show a spherical shape, which was identical to that of the initial minerals found in P6 epiphysis, despite their relatively small size (Fig. 4F). At pH 6.5 and $\mathrm{pH} 7.5$, the minerals presented a long ribbon-like and a short flake-like shape, respectively (Fig. 4E).

Together, these results not only indicate that alkaline $\mathrm{pH}$ is important for priming the cartilaginous environment for optimal phosphatase activity, but they also indicate that alkaline $\mathrm{pH}$ facilitates mineral precipitation even at low amounts of calcium and determines the spherical shape of the initial minerals.

\section{Alkaline milieu enhances bone tissue synthesis in vitro}

We then investigated the effect of $\mathrm{pH}$ on the synthesis of the extracellular matrix by chondrocytes. ATDC5 cells were cultured in micromasses under different $\mathrm{pH}$ conditions, and as shown in Fig. S1 (ESI $\dagger$ ), alkaline $\mathrm{pH}$ enhanced the synthesis of the cartilaginous extracellular matrix, stained with alcian blue. These results suggest that alkaline $\mathrm{pH}$ is associated with cartilage growth and maturation, and that alkaline $\mathrm{pH}$ promotes the synthesis of the cartilaginous matrix for mineral precipitation.

We have previously demonstrated that mechanical and osmotic pressure modulates chondrocyte burst, which in turn was associated with the origin of cell membrane nanofragments for mineral nucleation, and space-making for mineral expansion during initial mineralization of epiphysis at P6. ${ }^{1,17}$ To analyze the effect of alkaline $\mathrm{pH}$ on chondrocyte burst, entire femur epiphysis explants were maintained under different $\mathrm{pH}$ conditions and
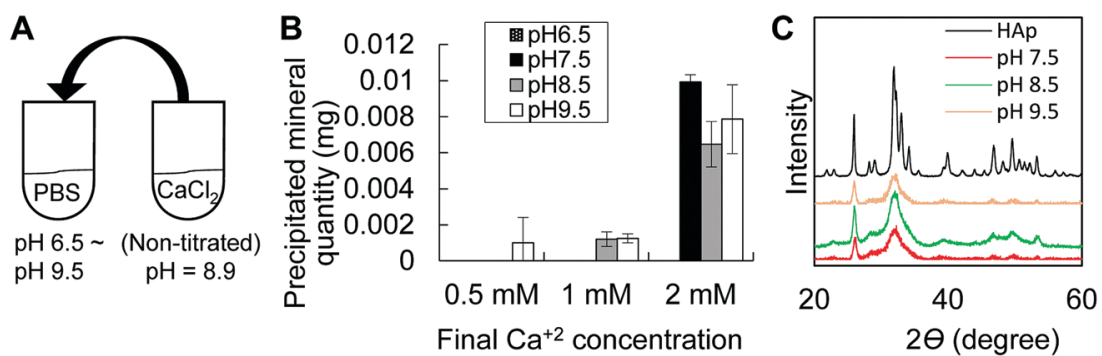

Fig. 3 In vitro mineral precipitation assay. (A) Schematic design of the experimental protocol. (B) Graph shows that an alkaline environment (pH 8.5 and $\mathrm{pH}$ 9.5) allows mineral precipitation at low concentrations of calcium solutions (0.5 mM or $1 \mathrm{mM}$ ). (C) XRD patterns showing peaks for HAp. Reference material is commercially available HAp. 

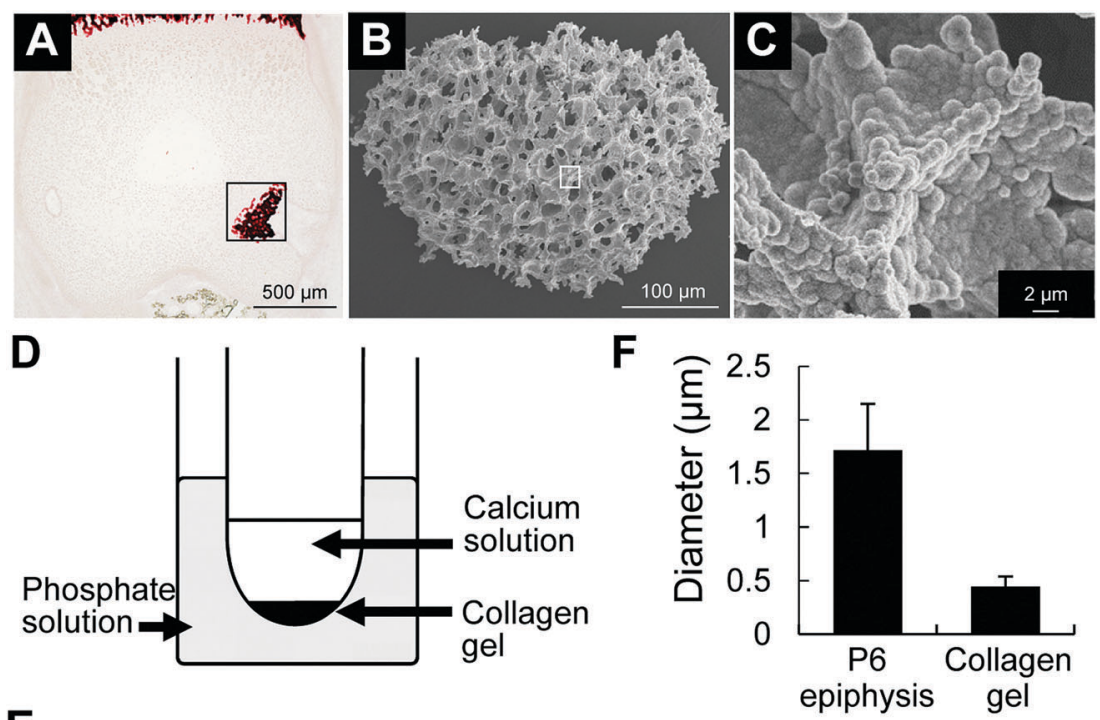

E
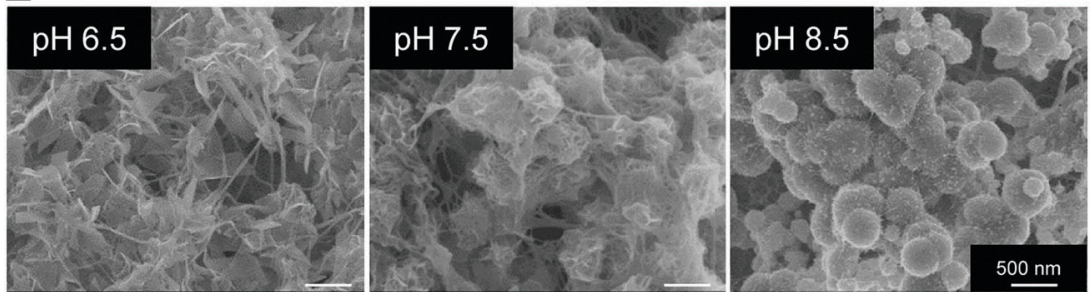

Fig. 4 FE-SEM observations of minerals formed in vivo and in collagen gel mineralization assay. (A) Alizarin red staining of histological section of P6 epiphysis. ( $B$ and $C$ ) FE-SEM images of minerals formed in vivo after sodium hypochlorite treatment. ( $B$ and $C$ ) High magnification images of the areas highlighted inside the squares in (A) and (B), respectively. (D) Schematic design of the experimental protocol using collagen gel, and calcium and phosphate solutions. (E) FE-SEM images of minerals formed in in vitro collagen gel mineralization assay. At pH 6.5, formation of ribbon-shape minerals. At pH 7.5, formation of short flake-like minerals. At pH 8.5, formation of spherical minerals. (F) Graph shows the comparative analysis of the size of spherical minerals formed in vivo and in vitro $(\mathrm{pH} 8.5)$.

observed under a time-lapse microscope (Fig. 5A). Analysis revealed that an alkaline environment $(\mathrm{pH} 8.5)$ induced a marked

increase in the number of chondrocyte bursts compared to $\mathrm{pH} 7.5$ or pH 6.5 (Fig. 5B). Next, the entire cartilage epiphysis explants
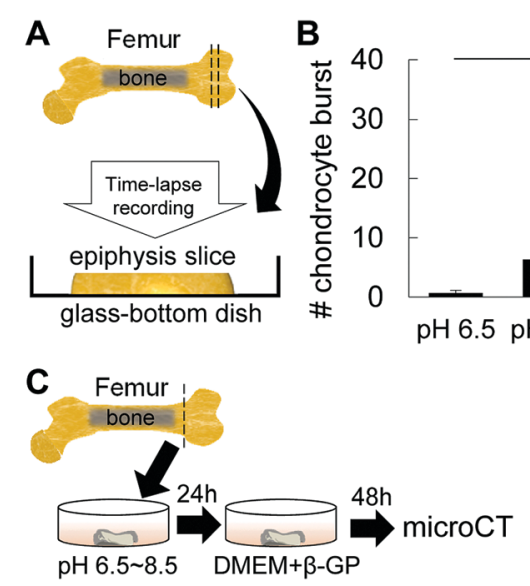
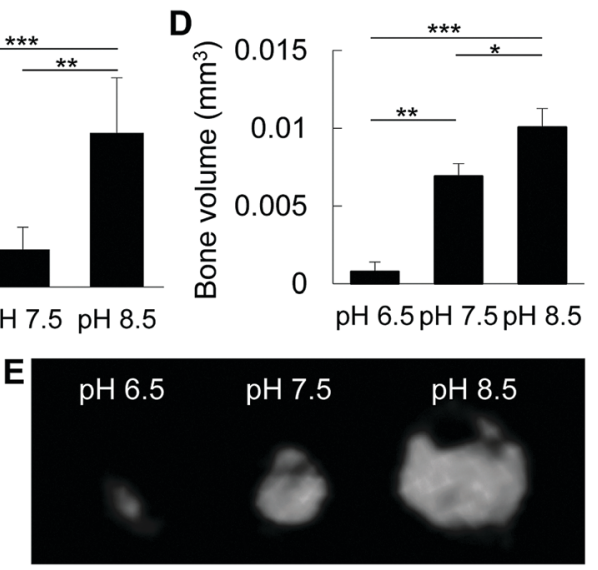

Fig. 5 Effect of $\mathrm{pH}$ on chondrocyte fate and mineral expansion. (A) Schematic design of the sample preparation for time-lapse analysis of chondrocyte burst. (B) Graph shows the number of chondrocyte bursts when epiphysis explants were maintained in culture media with different pHs. Note the markedly high number of chondrocyte bursts under alkaline conditions. (C) Schematic design of the sample preparation for analysis of bone formation in epiphysis after inducing chondrocyte burst by $\mathrm{pH}$ treatment. Epiphysis samples were maintained in culture media at different $\mathrm{pHs}$ for $24 \mathrm{~h}$ and then incubated for an additional 2 days in culture medium (D-MEM) supplemented with $\beta$-GP ( $\beta$-glycerophosphate). (D and E) Graph and micro-CT images of bone volume of P6 epiphysis explants after in vitro culture at different $\mathrm{pHs} .{ }^{*} p<0.05,{ }^{* *} p<0.01,{ }^{* *} p<0.001$, ANOVA, Tukey test. Data show mean \pm SD of at least four different samples. For (D), representative pictures of at least 4 different samples. 
were maintained in DMEM/F12 titrated to obtain different pHs (pH 6.5, pH 7.5 and $\mathrm{pH} 8.5$ ) temporarily for $24 \mathrm{~h}$ (Fig. 5C) and cultured in mineralization-inducing medium (DMEM/F12 supplemented with $\beta$-glycerophosphate) for an additional 2 days. As shown in Fig. 5C and D, the amount of bone formed after incubation in alkaline $\mathrm{pH}$ was significantly larger than that in pH 7.5 or pH 6.5, as determined by micro-CT analysis. Together, these results indicate that alkaline $\mathrm{pH}$ affects chondrocyte activity to prime the microenvironment for bone formation, and induces chondrocyte burst, which may be the origin of cell membrane nanofragments. ${ }^{1,17}$

Finally, to analyze the effect of $\mathrm{pH}$ on the mineralization of cell membrane nanofragments, we collected artificial nanofragments from cultured pre-chondrogenic ATDC5 cells, as reported. ${ }^{1}$ The artificial nanofragments were then incubated in $\alpha$-MEM supplemented with $\beta$-glycerophosphate in 48 well plates (Fig. 6A). Interestingly, mineralization of cell membrane nanofragments, which was reported to require 2 or 3 days, was strongly enhanced in alkaline $\mathrm{pH}$ compared to $\mathrm{pH} 7.5$, and occurred in just 1 day (Fig. 6B).

\section{Discussion}

The femur epiphysis starts to mineralize at P6, and this allows the observation of dynamic changes in organic and inorganic materials in a time- and stage-specific manner. Initial mineralization is based on chondrocytes only, i.e., there are no other cell types inside the epiphysis at P6. ${ }^{1,17}$ Therefore, we used the femur epiphysis as an in vivo model for analysis of the changes in the microenvironment, and we found that alkaline milieu is an important factor priming and determining initial mineralization at P6, with clear effects not only on cellular activities, but also on the precipitation and morphology of the minerals.

Our results indicated that alkaline phosphatase activity preceded mineral formation in a spatio-temporal manner. The importance of
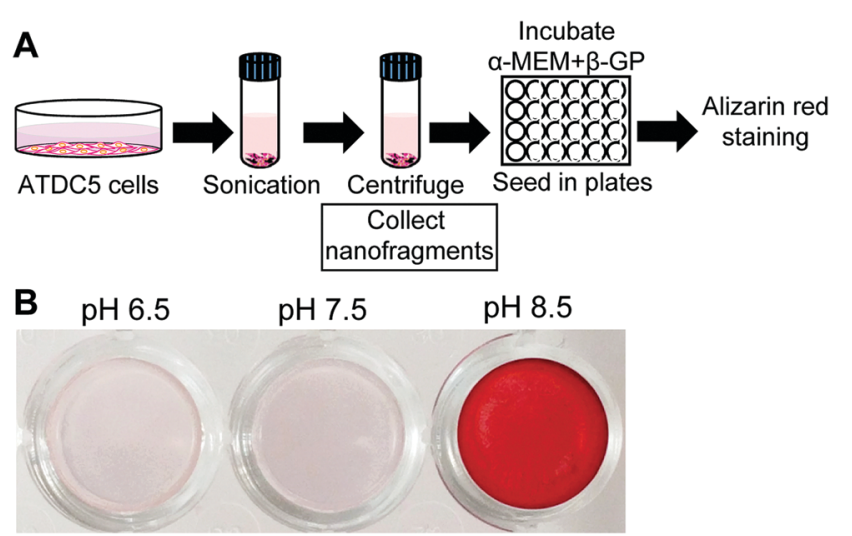

Fig. 6 In vitro mineralization using artificial chondrocyte membrane nanofragments. (A) Experimental protocol for artificial chondrocyte membrane isolation and mineralization. Artificial nanofragments were isolated after a series of centrifugation steps and then incubated in $\alpha$-MEM supplemented with $10 \mathrm{mM} \beta$-GP ( $\beta$-glycerophosphate). (B) Alizarin red staining showing chondrocyte membrane nanofragment mineralization in just 1 day, when the environmental pH is alkaline.
TNAP in mineralization has been demonstrated in previous animal models showing no mineralization in the epiphysis of 10-day-old TNAP knock-out mouse. ${ }^{31}$ TNAP is known to be mainly localized in the inner region of plasma membrane. ${ }^{32}$ Recently, we reported that plasma membrane nanofragments were the nucleation site for mineral formation. ${ }^{1}$ Therefore, the enzymatic activity of TNAP in catalyzing the plasma membrane phospholipids could promote the release of phosphate ions, which could subsequently bind to calcium to form the initial amorphous calcium-phosphate minerals, preferentially in an alkaline $\mathrm{pH}$. This is demonstrated by the rapid mineralization of cell membrane nanofragments in just one day, in alkaline conditions (Fig. 6).

The optimal $\mathrm{p} K_{\mathrm{a}}$ for alkaline phosphatase activity has been reported to be from 8.5 to $9.5 .^{22}$ On the other hand, a $\mathrm{pH}$ of 6.9 has been demonstrated to downregulate the mRNA levels of alkaline phosphatase. ${ }^{33}$ Therefore, the possibility of an alkaline environment promoting calcification in calcifying tissue has been hypothesized, but on the other hand, it has also been neglected due, in part, to the technical difficulty of sample preparation and accurate measurement of $\mathrm{pH}$ in calcifying tissues. For instance, a previous study showed that the $\mathrm{pH}$ in the femur epiphysis was approximately 7.5. ${ }^{23}$ Another study investigated the $\mathrm{pH}$ levels in freshly collected growth plate samples and found areas of relative alkalinity $(\mathrm{pH} 7.8-8.0)$ in matured chondrocytes, and of relative acidity ( $\mathrm{pH}$ 6.6-6.8) in hypertrophic chondrocytes and the calcifying zone. ${ }^{34}$ However, since $\mathrm{pH}$ measurement was performed with Lock's solution or molecular fluorescent probes, the effect of these solutions on pH cannot be neglected. In this study, the epiphysis was used as an in vivo tissue model for analysis of $\mathrm{pH}$ during initial mineralization. Its relatively soft and avascular characteristics allowed a clear and robust measurement of $\mathrm{pH}$ using a fluorescent sensor sheet. Measurement of the microenvironment using a pH sensor sheet confirmed the alkalinity of the epiphysis during initial mineralization, which would optimize the activity of TNAP.

Regarding the regulation of $\mathrm{pH}$ in vivo, several reports have pointed out carbonic anhydrases (CAs) as the major enzymes controlling the $\mathrm{pH}$ by the $\mathrm{CO}_{2}+\mathrm{H}_{2} \mathrm{O} \rightleftharpoons \mathrm{H}^{+}+\mathrm{HCO}^{3-}$ system. The non-enzymatic interconversions of $\mathrm{CO}_{2}$ and bicarbonate are known to be rather slow, and the existence of CAs has been predicted and their activity has been confirmed to be among the fastest of enzymes known. ${ }^{23}$ This enzyme also plays critical roles in controlling and accelerating $\mathrm{CaCO}_{3}$ deposition in vitro. ${ }^{35}$ Calcite deposition in the sponge system is decisively controlled enzymatically by CAII. Previous studies have demonstrated the expression of CAII in cartilage growth plate, ${ }^{36,37}$ and inhibition of CA activity with acetazolamide resulted in reduced rates of mineralization. ${ }^{38}$

Regarding the effect of alkaline $\mathrm{pH}$ on mineral nucleation, the major parameter that establishes the critical value for nucleation is known to be the solubility of ions. ${ }^{39}$ In fact, the solubility of calcium and phosphate ions was shown to be highly dependent on the solution $\mathrm{pH}^{40}$ The solubility of both $[\mathrm{Ca}]$ and $[\mathrm{P}]$ decreases at higher $\mathrm{pH},{ }^{40}$ indicating a higher tendency for calcium phosphate precipitation at higher $\mathrm{pH}$, even with lower amounts of [Ca]. 
Ion solubility is also decisive in determining mineral growth, as the high precipitability of $[\mathrm{Ca}]$ and $[\mathrm{P}]$ ions at high pH would favor calcium phosphate nucleation, whereas the low precipitability of these ions at low $\mathrm{pH}$ would favor calcium phosphate precipitation in the already existing minerals, subsequently promoting the growth of the crystals. Previous studies with hydroxyapatite have also demonstrated that $\mathrm{pH}$ is one significant factor affecting the structure and morphology of HAp. ${ }^{41}$ Under alkaline conditions, isotropic or weak-anisotropic growth occurs, i.e., the crystallites form spherical nanoparticles. However, under acidic conditions, anisotropic growth occurs, and the crystallites grow to form large rods or plates. ${ }^{41,42}$

In summary, the present results demonstrated that in vivo $\mathrm{pH}$ in femur epiphysis is alkaline, which is an important factor determining initial mineralization in femur epiphysis, by affecting not only the chondrocyte fate and synthesis of the extracellular matrix, but also by priming the environment for mineral precipitation, maturation and growth. Additionally, $\mathrm{pH}$ control of the microenvironmental condition could further allow the optimization of protocols for in vitro bone tissue synthesis, allowing the formation of bone-like tissue from cell-derived nanofragments in just one day.

\section{Author contributions}

E. S. H. designed and performed all experiments, collected and analyzed data, prepared figures and wrote the manuscript. M. O. designed part of the experiments and analyzed data. T. K. and T. N. supplied materials and equipment. T. M. designed part of the experiments, analyzed data, and wrote part of the manuscript.

\section{Data availability}

Data are available upon request to the corresponding author.

\section{Conflicts of interest}

The authors declare no competing financial interests.

\section{Acknowledgements}

This work was supported by JSPS KAKENHI (Grant Numbers JP18K17119, JP16H06990, JP16H05533, JP25220912, JP25293402 and JP26106718). E. S. H. acknowledges the financial support by the Kyoto Technoscience Center.

\section{References}

1 E. S. Hara, M. Okada, N. Nagaoka, T. Hattori, T. Kuboki, T. Nakano and T. Matsumoto, ACS Biomater. Sci. Eng., 2018, 4, 617-625.

2 J. Mahamid, A. Sharir, L. Addadi and S. Weiner, Proc. Natl. Acad. Sci. U. S. A., 2008, 105, 12748-12753.
3 A. R. Amini, C. T. Laurencin and S. P. Nukavarapu, Crit. Rev. Biomed. Eng., 2012, 40, 363-408.

4 S. Bose, S. Vahabzadeh and A. Bandyopadhyay, Mater. Today, 2013, 16, 496-504.

5 Y. Liu, D. Luo and T. Wang, Small, 2016, 12, 4611-4632.

6 K. Gkioni, S. C. G. Leeuwenburgh, T. E. L. Douglas, A. G. Mikos and J. A. Jansen, Tissue Eng., Part B, 2010, 16, 577-585.

7 H. Cölfen and M. Antonietti, Angew. Chem., Int. Ed., 2005, 44, 5576-5591.

8 M. P. Gashti, M. Stir and J. Hulliger, Colloids Surf., B, 2013, 110, 426-433.

9 K. Nakashima, X. Zhou, G. Kunkel, Z. Zhang, J. M. Deng, R. R. Behringer and B. De Crombrugghe, Cell, 2002, 108, 17-29.

10 T. Komori, H. Yagi, S. Nomura, A. Yamaguchi, K. Sasaki, K. Deguchi, Y. Shimizu, R. T. Bronson, Y. H. Gao, M. Inada, M. Sato, R. Okamoto, Y. Kitamura, S. Yoshiki and T. Kishimoto, Cell, 1997, 89, 755-764.

11 H. Aberle, H. Schwartz, H. Hoschuetzky and R. Kemler, J. Biol. Chem., 1996, 271, 1520-1526.

12 T. Maruyama, A. J. Mirando, C. X. Deng and W. Hsu, Sci. Signaling, 2010, 3, ra40.

13 B. Shu, M. Zhang, R. Xie, M. Wang, H. Jin, W. Hou, D. Tang, S. E. Harris, Y. Mishina, R. J. O'Keefe, M. J. Hilton, Y. Wang and D. Chen, J. Cell Sci., 2011, 124, 3428-3440.

14 R. E. Wuthier and G. F. Lipscomb, Front. Biosci., 2011, 17, 2812-2902.

15 H. C. Anderson, Clin. Orthop. Relat. Res., 1995, 266-280.

16 H. C. Anderson, J. Cell Biol., 1967, 35, 81-101.

17 E. S. Hara, M. Okada, N. Nagaoka, T. Hattori, L. M. Iida, T. Kuboki, T. Nakano and T. Matsumoto, Integr. Biol., 2018, 10, 57-66.

18 T. Matsumoto, M. Okazaki, M. Inoue, Y. Hamada, M. Taira and J. Takahashi, Biomaterials, 2002, 23, 2241-2247.

19 A. L. Boskey, B. D. Boyan, S. B. Doty, A. Feliciano, K. Greer, D. Weiland, L. D. Swain and Z. Schwartz, Bone Miner., 1992, 17, 257-262.

20 Y. Wang, T. Azaïs, M. Robin, A. Vallée, C. Catania, P. Legriel, G. Pehau-Arnaudet, F. Babonneau, M. M. Giraud-Guille and N. Nassif, Nat. Mater., 2012, 11, 724-733.

21 M. C. Yadav, A. M. S. Simão, S. Narisawa, C. Huesa, M. D. McKee, C. Farquharson and J. L. Millán, J. Bone Miner. Res., 2011, 26, 286-297.

22 J. L. Millán, Purinergic Signalling, 2006, 2, 335-341.

23 H. K. Vaananen, T. A. Hentunen and P. L. Härkönen, in The Carbonic Anhydrases, ed. Edwards Y. H., Chegwidden W. R. and Carter N. D., Birkhäuser, Basel, 2000, pp. 491-497.

24 E. S. Hara, M. Ono, H. T. Pham, W. Sonoyama, S. Kubota, M. Takigawa, T. Matsumoto, M. F. Young, B. R. Olsen and T. Kuboki, J. Bone Miner. Res., 2015, 30, 1585-1596.

25 M. Okada, A. Nakai, E. S. Hara, T. Taguchi, T. Nakano and T. Matsumoto, Acta Biomater., 2017, 57, 404-413.

26 H. Isaksson, M. J. Turunen, L. Rieppo, S. Saarakkala, I. S. Tamminen, J. Rieppo, H. Kröger and J. S. Jurvelin, J. Bone Miner. Res., 2010, 25, 1360-1366.

27 N. T. Khanarian, M. K. Boushell, J. P. Spalazzi, N. Pleshko, A. L. Boskey and H. H. Lu, J. Bone Miner. Res., 2014, 29, 2643-2652. 
28 E. S. Hara, M. Ono, Y. Yoshioka, J. Ueda, Y. Hazehara, H. T. Pham, T. Matsumoto and T. Kuboki, Cells Tissues Organs, 2016, 201, 88-96.

29 R. Yoh, T. Matsumoto, J. I. Sasaki and T. Sohmura, J. Biomed. Mater. Res., Part A, 2008, 87, 222-228.

30 E. S. Hara, M. Ono, S. Kubota, W. Sonoyama, Y. Oida, T. Hattori, T. Nishida, T. Furumatsu, T. Ozaki, M. Takigawa and T. Kuboki, Biochimie, 2013, 95, 374-381.

31 H. C. Anderson, J. B. Sipe, L. Hessle, R. Dhamyamraju, E. Atti, N. P. Camacho and J. L. Millán, Am. J. Pathol., 2004, 164, 841-847.

32 Y. Nakano, W. Beertsen, T. Vandenbos, T. Kawamoto, K. Oda and Y. Takano, Bone, 2004, 35, 1077-1085.

33 A. Brandao-Burch, J. C. Utting, I. R. Orriss and T. R. Arnett, Calcif. Tissue Int., 2005, 77, 167-174.

34 L. N. Wu, M. G. Wuthier, B. R. Genge and R. E. Wuthier, Clin. Orthop., 1997, 310-324.
35 W. E. G. Müller, H. C. Schröder, U. Schlossmacher, M. Neufurth, W. Geurtsen, M. Korzhev and X. Wang, FEBS Open Bio, 2013, 3, 357-362.

36 H. K. Vaananen, Histochemistry, 1984, 81, 485-487.

37 T. Kumpulainen and H. K. Väänänen, Calcif. Tissue Int., 1982, 34, 428-430.

38 G. R. Sauer, B. R. Genge, L. N. Y. Wu and J. E. Donachy, Bone Miner., 1994, 26, 69-79.

39 M. A. Meyers and P.-Y. C. Chen, Biological Materials Science Biological Materials, Bioinspired Materials, and Biomaterials, Cambridge University Press, 2014.

40 L. Chow, in Octacalcium Phosphate, ed. L. Chow and E. Eanes, Karger, Switzerland, 2001, pp. 94-111.

41 M. Sadat-Shojai, M. T. Khorasani, E. Dinpanah-Khoshdargi and A. Jamshidi, Acta Biomater., 2013, 9, 7591-7621.

42 F. G. O. Mekmene, S. Quillard, T. Rouillon, J.-M. Bouler and M. Piot, Dairy Sci. Technol., Springer, Netherlands, 2000, pp. 301-316. 